\title{
Géographie et production de l'espace: réflexions épistémologiques sur les rapports entre une science et la société
}

\section{Christian Vandermotten, Bruxelles}

\section{Introduction}

Le géographe soviétique KolossovsKI (1929-1951, éd. fr. 1975: 61) écrivait que

«L'histoire de l'évolution sociale peut être étudiée au travers de la géographie et, inversement, la géographie (économique) acquiert un caractère historique».

Il entendait par là les rapports entre la production historique et l'espace géographique. Mais on peut aussi, au second degré, considérer non pas l'espace géographique mais la science géographique, dans sa position sociale et dans les paradigmes qu'elle mobilise.

Cet article s'attachera à deux objectifs:

Le premier est d'examiner l'évolution des rapports entre la géographie (y compris ses premières expressions pré-scientifiques, ne visant pas à l'explication) et la société, sa nécessité sociale, qui en modèle les paradigmes dès qu'elle tente l'explication. Ceci n'exclut pas des moments d'autonomisation partielle du discours géographique par rapport aux besoins immédiats des forces sociales dominantes, moments portés par les logiques internes du monde et de la démarche scientifiques. Ces phases d'autonomisation ont pu conduire à des diminutions momentanées de l'intérêt accordé par ces forces sociales à la géographie, voire à l'émergence de paradigmes critiques, insérés eux aussi dans les réalités sociales, mais les examinant du point de vue de segments dominés.

Dans la mobilisation de ces paradigmes alternatifs, la géographie radicale occupe une position centrale depuis quatre décennies. S'appuyant sur sa critique positive, le second objet de cet article est de proposer une vision holistique de la géographie, science de la production spatiale.

\section{La place de l'espace dans les autres sciences}

L'espace occupe une place souvent marginalisée dans les sciences sociales, hors de la géographie (même, paradoxalement, chez certains géographes post-modernes, en particulier anglo-saxons): soit il est quasi absent, comme dans l'économie néo-classique, soit il se limite à être un réceptacle de l'investigation sociale ou histo- rique, un lieu où se passent des choses, sans examen de la dialectique entre les faits sociaux et la production spatiale. Même l'État est plus souvent perçu comme l'enveloppe politique, statistique dans laquelle sont envisagées les relations économiques, les phénomènes socio-politiques internes, les relations internationales, que comme un construit, un cadre d'interaction parmi d'autres, à des échelles plus grandes ou plus petites. Quant à la démarche marxiste classique, elle examine la nature de l'État, mais moins dans les spécificités de ses spatialités que dans son historicité (que sa vulgarisation réductrice et dogmatique inscrit dans un cheminement historique inéluctable).

Dans les sciences naturelles, l'espace occupe parfois une place, mais c'est un espace-contrainte ou un espace-milieu, hors du champ du social. En écologie, il est souvent vu comme une valeur en soi, attaquée par l'action des hommes, la subissant, même quand l'approche se prétend systémique.

\section{L'espace dans la géographie pré-scientifique}

La question épistémologique peut paraître non pertinente à propos de géographies qui ne visaient pas la compréhension des faits, mais seulement l'inventaire du monde et sa représentation, ne posant pas d'hypothèses, ne cherchant pas d'explication, du moins globale. L'examen de ces géographies pré-scientifiques est néanmoins intéressant: il apprend beaucoup sur le caractère non neutre de l'espace et de sa perception à un moment de l'histoire. On pourrait en dire autant d'une analyse historique des représentations paysagères.

Ainsi, dans le monde occidental médiéval et du début des Temps modernes, des descriptions plurielles de l'espace sont produites: cartes T-O, représentations normatives religieuses, découplées de la connaissance empirique du réel, qui disparaitront quand elles entreront en contradiction conflictuelle avec les réalités économiques et politiques, en premier lieu parce qu'elles affirment la platitude de la Terre; itinéraires, représentations linéaires à l'usage des marchands et pèlerins; portulans des marins, donnant les caps et une bonne représentation des côtes; plans terriers à grande échelle, qui se multiplieront avec la monétarisation croissante du tribut foncier (CEnTRe Pompidou 1980). Ces représentations traduisent la pluralité, le caractère non totalitaire de la société ouest-européenne de l'épo- 
que, ses rapports de pouvoir complexes entre seigneurs fonciers, Église, bourgeoisie marchande, pouvoir royal. Le dépassement de la crise de la fin du Moyen Âge par l'essor colonial et le mercantilisme imposera un système-monde dominé par ceux des pays européens où l'association entre bourgeoisie marchande et pouvoir d'État fut la plus efficace (BRAUDEL 1980). Le renforcement de l'État moderne s'accompagne à partir du $\mathrm{XVI}^{\mathrm{e}}$ siècle des débuts de sa cartographie et l'essor de l'économie-monde impose une représentation correcte des nouvelles routes et découvertes. Ceci impose l'intégration des codes de représentations cartographiques aux différentes échelles, une vision unifiée du monde. Le géographe-cartographe est alors un artisan et un homme d'affaires, lié aux marchands ou au Prince (VANDERmotTen 2008).

Avec le renforcement de l'État au XVIII ${ }^{\mathrm{e}}$ et au XIX ${ }^{\mathrm{e}}$ siècle, l'inventaire se systématise, devient statistique ou levé topographique exhaustif du territoire (GoLDLEWSKA 1993; VANDERMOTTEN 2008).

\section{Les cheminements de la géographie académique: déterminisme, possibilisme, néo-positivisme, behaviorisme}

La géographie scientifique s'individualise pour sa part dans le champ académique. Après une étape descriptive, classificatoire et naturaliste, teintée d'idéalisme chez Humboldt, ses débuts font la part belle au déterminisme génétique des conditions physiques, comme en France avec les chaînes causales d'ÉmiLE Levasseur (1872; Claval 1997a). Ce déterminisme dispense de l'examen des rapports sociaux, sinon chez un géographe «alternatif» comme Reclus (1905), qui les considère, mais d'un point de vue idéaliste, en n'évitant pas des remarques très déterministes, tout en reconnaissant la complexité du milieu et les capacités de l'homme de le dominer (VANDERMOTTEn 1986). Le phénomène présenté comme «naturel» apparaît incontestable et légitime le constat. Le charbon explique la révolution industrielle et justifie la suprématie britannique, sur les fondements et les méthodes de laquelle il ne faut dès lors plus s'interroger. Le milieu justifie le retard des régions tropicales; leur colonisation devient un acte de dévouement destiné à apporter la civilisation dans un environnement si difficile. L'explication de l'origine d'une industrie par la soi-disant qualité d'une ressource affirme de manière péremptoire celle de ses produits (VANDERMOTTEN 1979). Les sciences naturelles, auxquelles la géographie est initialement liée, surtout en Allemagne, découvrent l'évolutionnisme darwinien (Claval 2007; Ratzel 1882). L'extension du concept d'évolution au champ social conduit à la hiérarchisation des civilisations, légitime la colonisation, les positions de force des États.
Les outrances du déterminisme, son rejet partiel par la géographie française, liée à l'histoire et s'opposant à la science allemande, sa perte d'efficacité comme outil de promotion, conduisent à l'essor du possibilisme. Celui-ci s'exprime dans la multiplication de fines monographies et l'essor de la géographie régionale (VIDAL DE LA BLACHe 1888). Celle-ci ne fait pas référence à la dimension étatique, un donné non questionnable en France, à la différence de l'Allemagne, État de construction récente dont il convenait d'affirmer, comme le fera Haushofer (1934; 1941, éd. franç. 1986), la légitimité par le discours globalisant et impérialiste de la Geopolitik nationale-socialiste, qui s'articule avec les descriptions monographiques du Heimat local, fondement de «l'ancrage au sol» ratzélien (VANDERMOTTEN \& VANDEBURIE 2005).

Les démarches monographiques de la géographie régionale possibiliste sont donc caractérisées par l'oubli du politique et des rapports sociaux. La «nature naturelle» est un donné, dont l'importance s'efface devant les «genres de vie» (BRUNHEs 1910) ou, concept plus élaboré, la «civilisation» immanente (VIDAL DE LA Blache 1922) ou les iconographies (GotTMANN 1952), qui la modèlent à leur guise, en liaison avec les circulations. Gourou $(1969,1973,1982,1984)$ appliquera cette démarche à la géographie tropicale. En tout cas, les rapports entre la nature et les sociétés ne sont pas examinés dans une perspective dialectique. Sans réelle théorie, mue par l'ambition de battre le déterminisme en brèche à travers une suite de monographies, le possibilisme bute sur une impasse, malgré sa richesse informative. On retrouvera chez SAUER (1925) une situation similaire avec une certaine "géographie culturelle» idéaliste, où la culture est considérée comme un donné déterminant (Claval 1997b).

Après la Seconde Guerre mondiale, le fordisme, la consommation de masse, la banalisation des espaces effacent peu à peu les diversités chères aux régionalistes. La planification dans le cadre de la régulation keynésienne (destinée tant à contrer le communisme qu'à éviter au système capitaliste la répétition des impasses de l'avant-guerre) rend sans intérêt la poursuite de la répétition sans fin des monographies possibilistes, mal adaptées en outre à l'examen des problèmes urbains et industriels et dès lors de plus en plus cantonnées à des espaces ruraux résiduels ou «tropicaux». Cette géographie keynésienne de l'action est portée par Perroux (1950), qui dissocie l'espace économique du territoire physique et montre ses transformations permanentes, par Ponsard (1955) ou par Labasse (1966), seul ce dernier étant géographe de formation.

Dans ce contexte se développe aussi une géographie mathématisante; l'espace concret y disparaît au profit d'une abstraction géométrique, l'espace idéal de l'équi- 
libre spatial selon les prémisses a-sociales de l'économie néo-classique (IsARD 1956; Lösch 1940). La planification territoriale fordiste trouve aussi intérêt à cette géographie normative, technocratique et consensuelle. Ceux qui préfèrent des finalités plus pratiques à la finalité normative se cantonnent dans le behaviorisme (PRED 1967): l'espace y est seulement le cadre de l'enquête, sans interrogation sur les logiques qui dépassent la pseudo-rationalité d'individus isolés, sur les rapports avec le fonctionnement social global, ni sur la construction des représentations spatiales que ce fonctionnement entraîne.

\section{Du néo-positivisme à la géographie sociale radicale}

La géographie radicale s'affirme à partir de la fin des années soixante, en réaction politique aux présupposés de la géographie néo-positiviste ou au behaviorisme. Elle leur reproche de ne pas examiner le rôle premier des surdéterminations contraintes par les rapports sociaux, par les logiques du marché, de l'extraction et de l'appropriation de la plus-value, ni leur dialectique avec la production d'espaces qui sont à leur tour des facteurs influençant les modalités de la reproduction sociale.

Toutefois, cette géographie radicale entretient des rapports ambivalents avec les paradigmes néo-positivistes. Certes, elle s'en écarte résolument, en situant la formation des diversités et inégalités spatiales dans le cadre surdéterminant des rapports économiques et sociaux (Harvey 1974; Lipietz 1974, 1977; Castells 1975, ces deux derniers n'étant pas géographes). Elle ne considère pas les différenciations spatiales comme le produit «d'encrassements» historiques, que le libre jeu du marché et/ou des interventions volontaristes devraient faire disparaître, mais comme des conséquences inéluctables du jeu des rapports sociaux inégaux. A l'inverse du présupposé de la «rationalité» économique des acteurs, socialement neutres, des néo-positivistes, les géographes radicaux militent pour faire de la discipline un outil de compréhension de la différenciation de l'espace au service des luttes sociales, en particulier urbaines. Cependant, la géographie radicale se rapproche du courant néo-positiviste sur deux points:

- la mise en avant de «lois» de production de l'espace, universelles (du moins dans le cadre de l'économie capitaliste développée), et donc forcément réductrices de la réalité, même si elles la surdéterminent et rendent ici compte des rapports sociaux;

- la mobilisation de l'outil quantitatif, notamment les techniques de l'écologie factorielle, permise par le développement de l'informatique. En géographie urbaine, domaine de prédilection des radicaux, les résultats empiriques de ces analyses multivariées sont examinés à la lumière des modèles de l'École de Chicago (Kesteloot 1980).
Sans refuser, bien au contraire, le caractère surdéterminant des lois du capitalisme, il faut remarquer que ces apports majeurs à la compréhension de la différenciation spatiale ne suffisent pas à expliquer la variété des configurations spatiales, même au niveau de leur modélisation. La théorie de la rente foncière ne peut à elle seule expliquer pourquoi dans les villes belges ou anglo-saxonnes les pauvres sont plutôt concentrés en première couronne, alors que dans les villes françaises et méditerranéennes ils le sont plutôt en périphérie (VANDERMOTTEN et al. 1999). Elles ne prennent pas assez en compte les modalités spécifiques, locales du déploiement et de la régulation du système capitaliste, ni de ses articulations avec les modes de production préexistants. Or, celles-ci ne sont pas partout identiques, même entre des pays présentant des niveaux de développement économique similaires, voire à l'intérieur des cadres étatiques.

Intégrer ces spécificités ne résout d'ailleurs pas tout. Comprendre les variétés des configurations spatiales, tout en évitant de retomber dans la nomothétie monographique, impose la prise en compte simultanée de trois familles de facteurs: les événements factuels, les facteurs physiques et de localisation et les héritages culturels, tout en les replaçant, comme le fait la géographie radicale, dans le contexte historiquement déterminé des rapports sociaux.

\section{Du hasard et du behaviorisme au darwinisme social}

Il ne faut en effet pas rejeter la prise en considération d'événements factuels, tels que le hasard d'une initiative personnelle ou d'une découverte fortuite. Mais il s'agit d'examiner la portée de ces événements au prisme d'une sorte de darwinisme social, de leur tri sélectif dans l'histoire.

La découverte de l'Amérique par Colomb peut être un événement fortuit. Mais il s'inscrit dans un contexte global, celui de la soif de terres de la noblesse qui venait d'atteindre les limites de son développement extensif en Espagne même et des besoins en métaux précieux de l'État royal. Si ce n'eût été Colomb, un autre marin l'eût faite. Le contexte politique différent de la Chine, où la base de l'extorsion du surplus était une agriculture irriguée intensive, n'imposait pas les mêmes appétits coloniaux, voire les rendait dangereux pour la stabilité de la pyramide socio-politique: les expéditions maritimes chinoises restèrent sans suite. Le hasard existe, mais ses effets sont surdéterminés et socialement sélectionnés par les nécessités du système productif.

Cette sélection contribue à la différenciation et au modelage de l'espace. L'afflux de métaux précieux des 
Amériques dopera l'économie européenne en augmentant la disponibilité des liquidités (mais retardera celle de l'Espagne, dont il permet l'immobilisme des structures). Il en résultera une lente et inéluctable monétarisation des rapports sociaux et un renforcement compétitif des États ouverts sur l'Atlantique. Ce sera le terreau du mercantilisme, préalable au capitalisme industriel et à l'unification des marchés nationaux, et la base de la suprématie européenne pendant plusieurs siècles.

\section{Du déterminisme vulgaire au déterminisme dialectique historicisé}

La deuxième famille de facteurs significatifs relève du déterminisme dialectique historicisé, qui s'oppose au déterminisme vulgaire. Si ce dernier a été aisément démonté par la géographie possibiliste, celle-ci, faute d'une grille de lecture marxienne, en est arrivée à minimiser le rôle des facteurs physiques et de localisation. Elle les décrivait avec beaucoup de minutie, mais pour en refuser les déterminations qu'elle reportait sur des «civilisations» ou des «genres de vie» non autrement analysés dans leurs fondements. De la sorte, elle apparaît curieusement rejointe aujourd'hui dans le traitement du concept de «civilisation» par un HuntingTON (1997), dont elle était pourtant aux antipodes des conclusions à l'emporte-pièce, qui renvoient aux théories réalistes de la science politique, masquées derrière des argumentations pseudo-culturelles.

Ainsi, les géographes possibilistes vont nier l'impact de l'insularité de la Grande-Bretagne sur son développement précoce, au prétexte que Madagascar n'a pas connu de révolution industrielle. Mais le problème n'est pas là: il s'agit de resituer les avantages de l'insularité dans le cadre de formations sociales données, l'émergence des États mercantilistes et l'ouverture atlantique européenne. Ensuite, une systémique et une dialectique peuvent se construire entre les caractéristiques de la situation ou de la géographie physique, la formation sociale et l'évolution de sa spatialité. Dans un contexte d'essor des forces du capitalisme marchand, l'insularité britannique a pu contribuer au renforcement de la bourgeoisie, en maximisant son accès au commerce (et à la flibusterie) et en permettant à l'État de réduire ses dépenses militaires terrestres au profit d'une puissante flotte de guerre. On peut répéter le raisonnement à grande échelle, comme la situation et le site d'une ville et les différenciations socio-spatiales qui résultent de son usage, dans un contexte historique donné.

Cette vision du déterminisme dialectique historicisé peut encadrer un renouveau des rapports entre géographie physique et humaine, qui se nouent autour des questions environnementales et dont les liens avaient été rompus, tant par les néo-positivistes que par les radicaux, en réaction aux impasses du possibilisme, et par le repli des géographes physiciens sur l'étude des mécanismes dans une nature non socialisée. Si on considère que la société n'est pas une «civilisation» immanente, son fonctionnement, ses attitudes, son organisation politique rendent compte du niveau de développement des forces productives, des rapports sociaux de production, des conditions de l'appropriation des surplus. Mais cette production sociale peut être contrainte ou partiellement déterminée par la nature et ses conditions d'exploitation.

Mais toute nature est historicisée et n'est pas plus immanente; les contradictions entre société et nature débouchent sur des crises, économiques et/ou écologiques. Il faut se garder de les «naturaliser», de les ramener à des questions d'épuisement des ressources, sans les situer par rapport à leurs déterminants sociaux. Ce ne sont pas des épuisements «dans l'absolu». Faute de prendre ces précautions, on tombe dans des visions non dialectiques des crises (et des croissances, comme Rostow 1960), dans des perspectives néo-malthusiennes (manipulées par les forces économiques dominantes), s'appuyant sur des discours techniques, tels ceux du Club de Rome, les ramenant à une fatalité de la nature et de la disponibilité de ses ressources (MEADows et al. 1972). La crise écologique globale contemporaine n'est pas plus une crise dans l'absolu; elle est mondiale parce qu'elle reflète les impasses du système capitaliste lui-même globalisé. Les modalités politiques de son dépassement (à moins que le système n'implose) ne sont pas prévisibles. Ceci différencie une crise d'une fluctuation conjoncturelle. En tout cas, les solutions ne pourront plus être extensives comme lors de crises écologico-sociales antérieures: les limites du monde sont atteintes. Une telle approche dialectique réfute aussi les visions reclusiennes du retour à une soi-disant harmonie entre la nature et des communautés d'hommes libres, comme le mythe du bon sauvage de Rousseau (Reclus 1905). Si un tel équilibre originel avait existé, pourquoi s'en serait-on écarté?

\section{Temporalités différenciées, autoreproduction spatiale et hystérésis}

Refusant les soi-disant déterminismes marxiens (caricaturés à travers leurs vulgates) tout autant que les déterminismes physiques, la géographie culturelle et post-moderne entend pour sa part expliquer les caractères et la différenciation des espaces ou les comportements dans ceux-ci par les acquis culturels, les héritages, les mentalités, les préférences sociales, voire individuelles (Claval 2003; Mitchell 2000). Au pire, cela ramène certaines géographies à l'étude (ou plutôt à la description) des comportements (à peine) spatiali- 
sés de quelques individus, tendance fréquente dans la géographie anglo-saxonne. Mais ces facteurs restent largement inexplorés dans leurs formations et leurs spatialités. Certes, les mentalités, les attitudes sont de puissants déterminants des comportements des acteurs et donc de leurs impacts sur la production spatiale. Encore faut-il voir que ces mentalités et attitudes (et leurs représentations) sont loin d'être immuables.

Deux raisons au moins expliquent que l'historicité des mentalités et des attitudes n'apparaît pas à l'évidence. La première est que les forces sociales dominantes s'accommodent de cette présentation a-historique. Elle permet de neutraliser les discours critiques: les renouveaux régionaux dépendraient pour certains du seul changement des mentalités, qu'il suffirait de «vouloir» après en avoir décrété la nécessité, au prix évidemment d'une attaque frontale contre les acquis sociaux. La seconde est que la formation des mentalités résulte de processus complexes, difficiles à saisir, qui relèvent largement du non-écrit. Les histoires de ces formations peuvent être très longues et superposent des temporalités multiples. Cette complexité même dilue l'appréhension de leur historicité.

La complexité des processus est accentuée par l'importance des phénomènes d'hystérésis et d'autoreproduction sociale. L'hystérésis désigne un phénomène qui se poursuit, mais dont les causes objectives ont disparu. Les permanences des «mentalités» s'expliquent par leur autoreproduction sociale dans l'espace, à travers la famille et les diverses structures d'encadrement politico-sociales.

Si le plus souvent la temporalité des cultures et des mentalités est plus lente que celle des réalités économiques objectives, il peut aussi en aller autrement: outre le développement de l'instruction féminine, n'y a-t-il pas là une explication à la chute de la fécondité dans des pays de la périphérie, où les modèles culturels diffusés par les médias occidentaux anticipent sur les conditions objectives qui avaient déterminé dans les pays développés la lente baisse endogène de la fécondité?

\section{Classes sociales dominantes et dominées: une vision réductrice}

La géographie radicale (et bien plus encore la géographie catéchistique des pays du «socialisme réalisé») a aussi péché parfois par réductionnisme, ramenant exagérément les mécanismes aux seules logiques des intérêts de la classe dominante s'imposant à la classe dominée. C'est oublier qu'à l'intérieur de la classe dominante il existe des sous-groupes aux intérêts parfois conflictuels. C'est oublier aussi l'importance des classes intermédiaires, parfois objectivement alliées aux groupes dominants ou se projetant dans leurs valeurs, cherchant à d'autres moments à s'appuyer sur le mécontentement des groupes dominés, ainsi que la capacité des classes et fractions de classes dominantes à développer leur emprise idéologique sur de larges fractions des groupes intermédiaires et dominés, voire à nouer avec elles des alliances objectives.

Quelques exemples, à des échelles spatiales et temporelles différentes, illustreront ce propos:

On ne peut comprendre les succès de l'Europe à construire à son profit un système-monde sans considérer les alliances que de petits groupes de colonisateurs ont conclu, fut-ce momentanément, avec des fractions des groupes dominants ou intermédiaires locaux, euxmêmes en conflit avec d'autres fractions des groupes dominants locaux. De même, il est insuffisant de ramener, cinquante ans après la décolonisation, la responsabilité entière de la situation de l'Afrique à la colonisation, même si celle-ci en porte la responsabilité surdéterminante et si ses effets se prolongent à travers les mécanismes d'extraction de la plus-value contrôlés par les grandes firmes des pays du centre. Il ne faut pas pour autant négliger les liens objectifs entre cette responsabilité «en dernier recours» et la para-bourgeoisie étatique compradore au pouvoir dans les pays africains, groupe à la fois dominant à l'échelle nationale et intermédiaire à l'échelle mondiale.

On ne peut pas plus comprendre les réussites initiales, mais aussi les dérives bureaucratiques rapides des révolutions «socialistes» sans considérer la place dominante prise par les groupes intermédiaires, tels ceux issus de la petite bourgeoisie intellectuelle brimée matériellement et culturellement par les conséquences des retards de développement ou de la colonisation. Ce sont ces groupes intermédiaires qui, à travers une appropriation collective de l'appareil d'État, vont se substituer, au nom du prolétariat, à la bourgeoisie défaillante et mener le processus d'accumulation industrielle, en s'attaquant aux agrariens (ou à leur substitut colonial, comme en Algérie), mais en pressurant au passage la paysannerie de manière plus dure encore que ne l'avait fait la bourgeoisie des pays du centre aux premiers temps de la révolution industrielle.

A l'échelle intra-urbaine, bien des phénomènes ayant un impact sur la géographie ne peuvent se comprendre sans considérer les alliances complexes entre groupes et sous-groupes dominants, intermédiaires, voire dominés. Cela ne signifie pas que la proximité spatiale puisse devenir à elle seule un moteur des luttes urbaines et assurer leur caractère révolutionnaire à travers la formation de larges alliances fondées sur le terrain, comme la question fut posée dans les débats entre 
Lefebvre (1974) et CASTElls $(1972,1975)$. D'une part les «luttes urbaines» dominées par les classes moyennes intellectuelles durant les années 1970 au nom de la préservation d'une identité fondée sur des lieux, même quand elles prétendaient défendre le droit des classes populaires à la ville, relevaient de fait d'une défense de leur position historique relative, menacée par le fonctionnalisme technocratique résultant des logiques du grand capital et du développement fordiste. Ces groupes sociaux intermédiaires prolongent aujourd'hui ces luttes pour «l'urbanité», mais dans la société post-fordiste leur alliance principale a changé et se fait maintenant, dans le contexte de la gentrification, avec des fractions de la bourgeoisie d'affaires et de la bureaucratie internationale, voire du capitalisme immobilier, en partie reconverti vers le résidentiel auquel il avait préféré le bureau. D'autre part les révoltes urbaines violentes, qui sont le fait exclusif de fractions des classes dominées, relèvent soit de conflits mettant en présence des groupes ethniques dominés concurrents, soit d'événements s'apparentant plutôt aux jacqueries, sursauts de violence contre toutes les marques du pouvoir ou de la propriété. Dans la mesure où elles sont le fait de groupes marginalisés sans organisation politique et en outre, à la différence des paysans de l'Ancien Régime, non centraux dans le fonctionnement du système économique et sans relais parmi les groupes intermédiaires, ces révoltes sont vite maîtrisées par le pouvoir, au prix de quelques mesures sécuritaires ou superficielles, quand il ne les récupère pas politiquement à son profit.

La non-transformation des luttes urbaines en processus «révolutionnaire» tient au moins à deux raisons: d'abord, les différentes classes sociales sont très ségrégées dans l'espace; ensuite, même quand elles sont réunies en un même lieu, elles peuvent réagir différemment à un problème identique. Ainsi, pour appréhender les comportements sociaux des acteurs dans le champ de la géographie urbaine et leurs attitudes par rapport à la revitalisation des villes, il faut prendre en considération la complexité des articulations entre groupes et sous-groupes sur le terrain concret, y compris les «nouvelles élites» émergeant à l'intérieur des groupes dominés, leurs intérêts objectifs et leurs assimilations subjectives.

\section{Conclusions}

Une approche par la production sociale des espaces (Figure 1) dépasse le caractère parfois trop mécaniste de la géographie radicale «classique», sans en renier les apports majeurs. Elle permet, par l'examen de la dialectique entre les logiques complexes et contradictoires des groupes d'acteurs, des stratégies et alliances des forces sociales dominantes et intermédiaires (voire, dans une certaine mesure, dominées), de la prise en compte de l'héritage des différentes strates plus ou moins conservées des productions sociales et spatiales antérieures, d'accorder un rôle important aux superstructures ainsi qu'à la nature, non dans ses soi-disant déterminismes vulgaires mais dans son historicité. L'approche par la production sociale de l'espace ne néglige pas le culturel, le rôle des «mentalités», mais elle considère ces superstructures comme des produits mouvants de l'histoire. Le géographe doit aussi s'approprier les explications de leur formation. Elles peuvent acquérir une certaine autonomie, influencer le développement des infrastructures et le cours des évolutions spatiales dans la mesure où leur temporalité peut différer de celle des déterminants matériels «objectifs». L'approche est ainsi génétique en même temps qu'elle s'attache à l'analyse du fonctionnement de l'espace contemporain (ou de tout espace dans le passé - rompons avec cette affirmation sans consistance épistémologique que le champ de l'historien serait le passé et celui du géographe le présent).

L'approche transcende les disputes stériles entre déterminisme et possibilisme. Elle réconcilie théorie et empirie, quantitatif et qualitatif. Les changements d'échelle n'apparaissent plus comme des choix subjectifs du niveau d'étude, mais comme des angles d'approche articulés, éclairant les facettes d'une même réalité globale complexe. Géographie sociale et physique peuvent aussi retrouver des articulations, à travers la dialectique entre les formations sociales et la nature historicisée, qui débouche sur les enjeux environnementaux.

Les espaces doivent se lire comme les produits de successions de strates. Les plus anciennes, moins bien conservées, mais pouvant néanmoins freiner les évolutions récentes ou laisser des traces significatives, sont les produits de systèmes productifs et de rapports sociaux antérieurs. Ces strates ne sont pas que matérielles. Elles sont aussi constituées de permanences culturelles, de superstructures, qui déterminent pour une part les comportements des acteurs actuels, qu'il faut expliquer sous peine de tomber dans la vaine incantation au «changement des mentalités». Ces strates traduisent les actions d'acteurs qui ont agi à différentes échelles et les rapports de force entre ces acteurs et ces échelles d'action: dans la cellule familiale, les organisations locales, les structures régionales, nationales, multinationales. Il ne faut pas sous-estimer les impacts des alliances entre fractions de classes, mais l'espace est évidemment plus modelé par les acteurs et agents dominants que par les dominés. Ceci est d'autant plus perceptible que l'on travaille à plus petite échelle. D'où le risque de ne rien comprendre en se contentant de la monographie isolée à grande échelle. Les espaces ainsi produits, aires ou places, sont aussi les nœuds de réseaux de flux, eux aussi multidimensionnels. Ces 


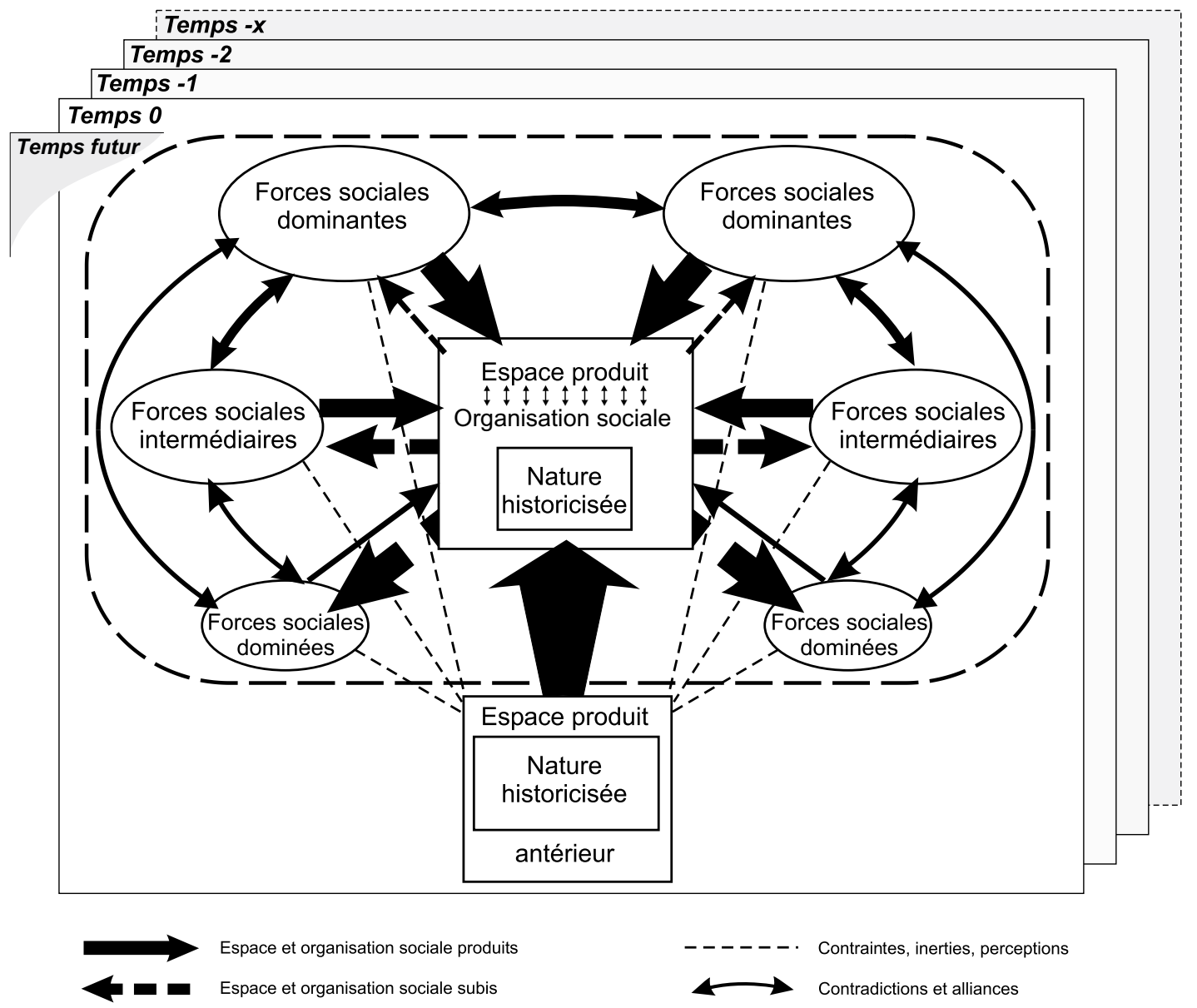

Fig. 1: La production sociale de l'espace

The social production of space

Die soziale Produktion des Raumes

Source: VANDERmotTen \& MARISSAL 2003-2004

réseaux et les informations qui y sont véhiculées sont aussi des facteurs majeurs de la production spatiale, mais il ne faut pas tomber dans le piège de les considérer comme des lieux de pouvoir «en eux-mêmes», comme souvent dans la géographie des réseaux, de la même manière qu'en géographie politique l'État est souvent perçu comme un lieu de pouvoir «en soi», sans analyser sa nature.

Il s'agit en fin de compte, pour comprendre le monde, de construire une «simple et hétérodoxe science sociale géohistorique» (TAYLOR 1996), transcendant les corporatismes disciplinaires, de pratiquer une «géohistoire» (Grataloup 2007). L'intégration des formations cultu- relles et des superstructures doit se faire dans une démarche géographique dialectique et matérialiste, comme dans l'enrichissement gramscien de la démarche marxiste. Cela renvoie aussi à BRAUDEL (1980) et à WALLERSTEIN (1980-1985), à TAYLOR (1985) aux temps longs et à l'imbrication des diverses temporalités, à l'histoire comme nécessité pour la géographie et à l'espace concret (certainement non réductible aux «contraintes naturelles») comme élément indispensable à la compréhension des processus historiques. Ou encore aux conceptions de SANTOS (1985) sur la dialectique entre les forces sociales agissant dans et sur l'espace et la nature historicisée, à la totalité du réel structurant l'espace à travers le jeu de techniques elles-mêmes historicisées. 


\section{Bibliographie}

BRAUdel, F. (1980): Civilisation matérielle, économie et capitalisme. - Paris: Armand Colin.

BrunheS, J. (1956): La géographie humaine. - Paris: Presses Universitaires de France.

Castells, M. (1972): La question urbaine. - Paris: Maspero.

CASTElls, M. (1975): Luttes urbaines et pouvoir politique. - Paris: Maspero.

Centre Pompidou (1980): Cartes et figures de la Terre. - Paris.

Claval, P. (1997a): L'évolution de quelques concepts de base de la géographie. Espace, milieu, région, paysage (1800-1900). - In: STASZAK, J.F. (dir.): Les discours du géographe. - Paris: L'Harmattan: 89-118.

Claval, P. (1997b): La géographie culturelle et l'espace. - In: StaszaK, J.F. (dir.): Les discours du géographe. - Paris: L'Harmattan: 119-144.

Claval, P. (2003): Géographie culturelle. Une nouvelle approche des sociétés et des milieux. - Paris: Armand Colin.

Claval, P. (2007): Géographies et géographes. - Paris: L'Harmattan.

GoldLEWSKA, A. (1993): La géographie des ingénieurs géographes (1795-1830). - In: Claval, P. (dir.): Autour de Vidal de la Blache. La formation de l'école française de géographie. - Paris: Centre national de la recherche scientifique (CNRS) Éditions: 29-35.

Gotrmann, J. (1952): La politique des États et leur géographie. - Paris: Armand Colin.

Gourou, P. (1969): Recueil d'articles. - In: Revue belge de géographie 93, 1-2-3: 3-450.

Gourou, P. (1973): Pour une géographie humaine. - Paris: Flammarion.

Gourou, P. (1982): Terres de bonne espérance. Le monde tropical. - Paris: Plon.

Gourou, P. (1984): Riz et civilisation. - Paris: Fayard.

Grataloup, C. (2007): Géohistoire de la mondialisation. - Paris: Armand Colin.

Harvey, D. (1974): Class-monopoly, rent, financial capital and the urban revolution. - In: Regional Studies 8, 3-4: 239-255.

HAUSHOFER, K. (1934): Weltpolitik von heute. - Berlin: Verlag Zeitgeschichte.

Haushofer, K. (1941, éd. franç. 1986): De la géopolitique. - Paris: Fayard.

Huntington, S. (1997): Le choc des civilisations. - Paris: Odile Jacob.

IsARD, W. (1956): Location and space-economy: a general theory relating to industrial location, market areas, land use, trade and urban structures. - Cambridge: The MIT Press.

Kesteloot, C. (1980): De ruimtelijke sociale struktuur van Brussel-Hoofdstad. Kritische evaluatie van de faktoriële ekologie en alternatieve kijk op de stad. - = Acta Geographica Lovaniensia 19, Katholieke Universiteit Leuven.
Kolossovski, N. (1929-1951, éd. fr. 1975): Théorie de la régionalisation économique. - Moscou: Éditions du Progrès.

Labasse, J. (1966): L'organisation de l'espace. - Paris: Hermann.

Lefebvre, H. (1974): La production de l'espace. - Paris: Anthropos.

Levasseur, E. (1872): L'étude et l'enseignement de la géographie. - Paris: Delagrave.

LiPIETZ, A. (1974): Le tribut foncier urbain. - Paris: Maspero.

LiPIETZ, A. (1977): Le capital et son espace. - Paris: Maspero.

Lösch, A. (1954): The economics of location. - New Haven: Yale University Press.

Meadows, D., Randers, J. \& W. Behrens (1973): Halte à la croissance? Rapport sur les limites de la croissance. - Paris: Fayard.

Mitchell, D. (2000): Cultural Geography: a critical introduction. - Oxford: Blackwell.

Perroux, F. (1950): Les espaces économiques. - In: Economie appliquée 3, 1:225-244.

Ponsard, C. (1955): Economie et espace. - Paris: Société d'édition d'enseignement supérieur (SEDES). Pred, A. (1967): Behaviour and location. - = Lund Studies in Geography B 29.

RAtZel, F. (1882): Anthropogeographie oder Anwendung der Erdkunde auf die Geschichte. - Stuttgart: J. Engelhorn.

Reclus, E. (1905): L'Homme et la Terre. - Paris: Librairie Universelle.

Rostow, W.W. (1960): The stages of economic growth: a non-communist manifesto. - Cambridge: Cambridge University Press.

SANToS, M. (1989): Espace et méthode. - Paris: Publisud. SAUER, C.O. (1925): The morphology of landscape. - In: University of California Publications in Geography 2, 2: 19-53.

TAYLOR, P.J. (1985): Political Geography. World-economy, nation-state and locality. - Harlow: Prentice Hall. TAYLOR, P.J. (1996): On the nation-state, the global and social science. - In: Environment and Planning A 28, 11: 1917-1995.

VANDERMotTen, C. (1979): Pour une macrogéographie industrielle. 200 ans de pensée en géographie industrielle. L'exemple de la Belgique. - In: Revue belge de Géographie 103, 1: 3-67.

VANDERMOTten, C. (1986): La pensée d'Elisée Reclus et la géographie de la Belgique en son temps. - In: Revue belge de Géographie 110,1:71-94.

VANDERMOTTEN, C. (2008): L'histoire de la géographie belge à travers les biographies nationales. - In: Belgeo 9,1:105-122.

Vandermotten, C., Vermoesen, F., De Lannoy, W., De Corte, S. et al. (1999): Villes d'Europe. Atlas comparatif. - In: Bulletin trimestriel du Crédit Communal de Belgique 53, 1-2:1-408. 
VANDermotten, C. \& P. Marissal (2003-2004): La production des espaces économiques. - Bruxelles: Éditions de l'Université de Bruxelles.

VAndermotten, C. \& J. VAndeburie (2005): Territorialités et politique. - Bruxelles: Éditions de l'Université de Bruxelles.

Vidal de la Blache, P. (1888): Des divisions fondamentales du sol français. - Paris: Bulletin littéraire 2: 129-142.

Vidal de la Blache, P. (1922): Principes de géographie humaine. - Paris: Armand Colin.

WALLERSTEIN, I. (1980-1985): Le système-monde du $\mathrm{XV}^{\mathrm{e}}$ siècle à nos jours. - Paris: Flammarion.

\section{Résumé: Géographie et production de l'espace: réflexions épistémologiques sur les rapports entre une science et la société}

Partant d'une analyse critique des rapports entre les différentes formes et écoles de la géographie et l'évolution socio-économique, l'article propose un approfondissement de la géographie radicale classique, en y intégrant les apports d'un déterminisme géographique historicisé, qui n'exclut en rien le rôle essentiel des constructions culturelles et des superstructures. La démarche débouche aussi sur une réunification de la géographie sociale et de certaines approches de la géographie physique.

Mots-clés: épistémologie, histoire de la géographie, géographie radicale, production sociale de l'espace

\section{Summary: Geography and the production of space: epistemological observations on the relation between science and society}

Starting from a critical reflection on the relation between geography, geographic schools and the socio-economic evolution, the paper emphasizes the necessity to go beyond «classical» radical geography and to include the contributions made by historicized geographical determinism. This approach allows the inclusion of aspects such as cultural buildings and other superstructures. The process is argued to lead to a reunification of social geography with some approaches in physical geography.

Keywords: epistemology, history of geography, radical geography, social production of space

\section{Zusammenfassung: Geographie und Produktion des Raums: epistemologische Überlegungen zu den Beziehungen zwischen einer Wissenschaft und der Gesellschaft}

Ausgehend von einer kritischen Analyse der Beziehungen zwischen den verschiedenen Schulen der Geographie und der sozioökonomischen Entwicklung, bietet der Artikel eine Vertiefung der klassischen radikalen Geographie: Beiträge eines historisierenden geographischen Determinismus werden integriert, ohne dabei die wichtige Rolle kultureller und superstruktureller Konstruktionen auszuschliessen. Das Vorhaben eröffnet den Weg zu einer erneuten Zusammenführung von Sozialgeographie und einigen Ansätzen der Physiogeographie.

Schlüsselwörter: Epistemologie, Geschichte der Geographie, radikale Geographie, soziale Produktion des Raums

Prof. Dr. Christian Vandermotten, Université Libre de Bruxelles, Académie Royale de Belgique, Institut de Gestion de l'Environnement et d'Aménagement du Territoire, avenue F.D. Roosevelt, 50, CP 246, B-1050 Bruxelles, Belgique. e-mail:cvdmotte@ulb.ac.be

\section{Manuskripteingang/received/manuscrit entré le} 1.7.2008

Annahme zum Druck/accepted for publication/accepté pour l'impression: 20.11 .2008 